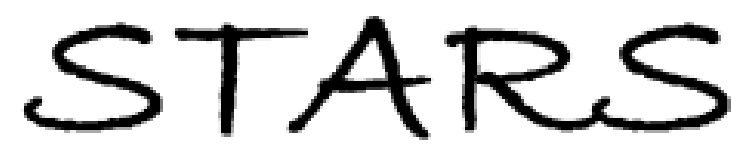

University of Central Florida

STARS

Faculty Bibliography 1990s

Faculty Bibliography

$1-1-1992$

\title{
Phase Controlled All-Optical Switching In Rocking Filter Fibers
}

C. G. Krautschik

University of Central Florida

G. I. Stegeman

University of Central Florida

R. H. Stolen

Find similar works at: https://stars.library.ucf.edu/facultybib1990

University of Central Florida Libraries http://library.ucf.edu

This Article is brought to you for free and open access by the Faculty Bibliography at STARS. It has been accepted for inclusion in Faculty Bibliography 1990s by an authorized administrator of STARS. For more information, please contact STARS@ucf.edu.

\section{Recommended Citation}

Krautschik, C. G.; Stegeman, G. I.; and Stolen, R. H., "Phase Controlled All-Optical Switching In Rocking Filter Fibers" (1992). Faculty Bibliography 1990s. 504.

https://stars.library.ucf.edu/facultybib1990/504

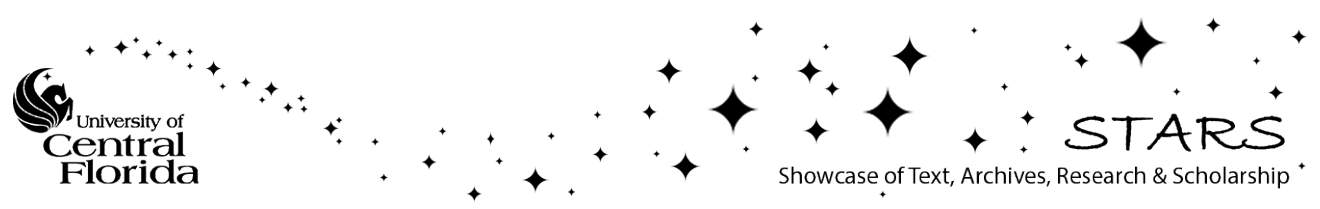




\section{Phase controlled all-optical switching in rocking filter fibers}

Cite as: Appl. Phys. Lett. 61, 1751 (1992); https://doi.org/10.1063/1.108416

Submitted: 22 May 1992 . Accepted: 30 July 1992 . Published Online: 04 June 1998

C. G. Krautschik, G. I. Stegeman, and R. H. Stolen

\section{ARTICLES YOU MAY BE INTERESTED IN}

Demonstration of demultiplexing with a rocking filter fiber

Applied Physics Letters 63, 860 (1993); https://doi.org/10.1063/1.109881

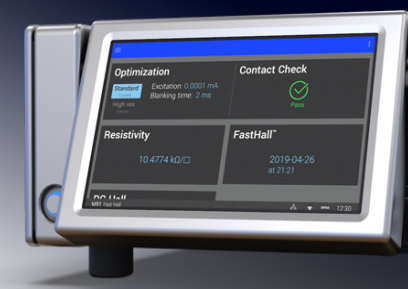
M91 FastHall ${ }^{\text {TM }}$ Controller

A revolutionary new instrument for complete Hall analysis See the video 0 


\title{
Phase controlled all-optical switching in rocking filter fibers
}

\author{
C. G. Krautschik and G. I. Stegeman \\ CREOL, University of Central Florida, 12424 Research Parkway, Orlando, Florida 32826
}

\author{
R. H. Stolen \\ AT\&T Bell Laboratories, Holmdel, New Jersey 07733
}

(Received 22 May 1992; accepted for publication 30 July 1992)

\begin{abstract}
We demonstrate experimentally that all-optical switching of a strong beam can be controlled by the phase of a weak beam in a rocking rotator fiber.
\end{abstract}

All-optical switching in fiber and channel waveguides relies on a high intensity beam to produce index changes via an intensity-dependent refractive index. This index change leads to switching between two spatially or temporally separated outputs of the high power beam, or of a coincident weaker signal beam. ${ }^{1-8}$ In all of the experiments reported to date it is the intensity of the switching beams which is adjusted to produce different outputs and the optical phase at the device input has played no role. There has, however, been a proposal to switch a cw signal input between the two output channels of a nonlinear directional coupler (NLDC) by changing the phase by $\pi$ of a weak control beam injected into the second input channel. ${ }^{9}$ This requires a cw input signal just at the critical power for a NLDC. Such phase-controlled switching has not been observed, probably because most NLDCs are operated with pulse inputs which contain a continuous range of powers. In this letter we demonstrate for the first time a different form of phase-controlled switching in a nonlinear rocking filter fiber (NLRFF) which operates with pulse inputs. Here a phase change of approximately $\pi / 2$ in a simultaneously incident weak beam is shown to switch a strong signal (input into the orthogonal polarization) between the two output polarizations of a NLRFF.

A rocking filter fiber is an element which rotates the plane of polarization with distances down a fiber, a distance $L_{c}$ yields a net rotation of $\pi / 2 .{ }^{10}$ For optimum rotation, the periodic twist $\left(L_{t}\right)$ introduced during the pulling process equals the fiber birefringence beat length $\left(L_{b}=2 \pi\right.$ / $\left.\left|\beta_{x}-\beta_{y}\right|\right)$ at the operating wavelength. Increasing the input intensity produces a distance-dependent beat length of the form $2 \pi /\left|\left(\beta_{x}-\beta_{y}\right)+0.333 \gamma\left[P_{x}(z)-P_{y}(z)\right]\right|$, where $\gamma$ $=n_{2} \omega / c A_{\text {eff }}, n_{2}$ is the intensity dependent refractive index coefficient and $A_{\text {eff }}$ is the effective fiber cross-sectional area. As a result $L_{r} \neq L_{b}$ and the spatial rate of polarization rotation is slowed down or, at high powers, effectively stopped. Thus, for incident light polarized along, for example, the $x$ axis, the output polarization changes from $y$ to $x$ with increasing input power for a fiber $L_{c}$ long.

The equations governing the nonlinear evolution of the two polarization states (normalized amplitudes $b_{x}$ and $b_{y}$ ) for $L_{h}=L_{t}$ are

$$
\begin{aligned}
& -i \frac{d b_{x}}{d z}=\frac{\pi}{2 L_{c}} b_{y}+\frac{\gamma}{3}\left|b_{x}\right|^{2} b_{x}, \\
& -i \frac{d b_{y}}{d z}=\frac{\pi}{2 L_{c}} b_{x}+\frac{\gamma}{3}\left|b_{y}\right|^{2} b_{y} .
\end{aligned}
$$

The power-dependent response for the mode amplitudes is the same as that for the well-known NLDC, but the critical power $\left(P_{c}=3 \lambda A_{\text {eff }} / n_{2} L_{c}\right)$ for switching is 3 times larger than for the NLDC. Switching, both on $\left(L_{b}=L_{t}\right)$ and off $\left(L_{b} \neq L_{t}\right)$ filter resonance, has been demonstrated in NLRFFs and found to agree well with theoretical predictions. $^{3}$

We have explored numerically the solutions to these equations for the case where we seed the second polarization input with $b_{y}(0)=R b_{x}(0) e^{i \Delta \phi}$, where both $R$ and $\Delta \phi$ can be varied. Shown in Fig. 1 is the "bar" state output $\left[b_{y}\left(L_{c}\right)\right]$ when $10 \%$ of the incident power is used to "seed" the second polarization. Gaussian input pulses were assumed. The key result is that the output can be switched from $70 \%-80 \%$ to $5 \%-10 \%$ by changing the relative input phase of the seed beam from 0 to $\approx-\pi / 2$.

An interesting feature is that the response curves are not symmetric about $\Delta \phi=0$. This asymmetry can be understood directly from the equations which, in the absence of nonlinearity and seed beam, predict a define $(\pi / 2)$ phase relationship between the two polarizations. By changing the input phase of the seed beam $b_{y}(0)$ from $-\pi / 2$ to $\pi / 2$, the initial polarization conversion from $b_{x}(z)$ into $b_{y}(z)$ changes from being in phase to out of phase with the seed beam at the input end. That is, seeding in phase or out of phase with the initial growth of $b_{y}(z)$ effectively decreases or increases the total rotation achieved at the end of the fiber.

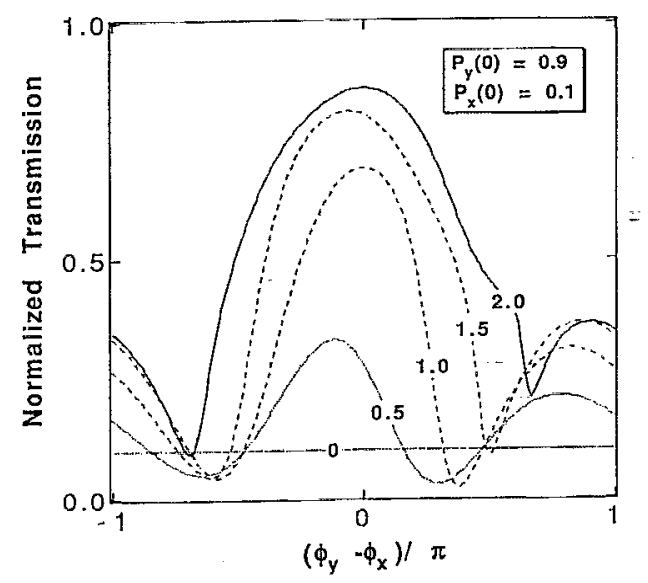

FIG. 1. Calculations of the phase controlled $\left[\Delta \phi=\left(\phi_{y}-\phi_{x}\right) / \pi\right]$ alloptical switching of the bar state, with $10 \%$ of the input power in the control pulse (cross state). The five different curves correspond to successive larger input powers of $0.00,0.5,1.0,1.5$, and $2.0 \mathrm{KW}$. 


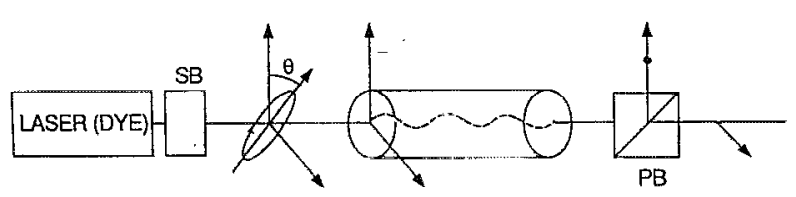

FIG. 2. Schematic showing how the seeded input into the fiber is obtained.

The theoretical predictions just discussed were tested experimentally with a nonideal fiber. The wavelength response of the rocking filter fiber used showed two distinct peaks, a maximum polarization rotation at $\lambda \simeq 590 \mathrm{~nm}$ and a smaller rotation (about $50 \%$ of the main peak) at 600 $\mathrm{nm}$. For $\pi / 2$ rotation at $590 \mathrm{~nm}$ the fiber was $185-\mathrm{cm}$ long with a birefringent beat length of $1.5 \mathrm{~cm}$. Taking into account the section centered at $600 \mathrm{~nm}$, the effective $L_{c}$ was $127-\mathrm{cm}$ long. Furthermore, the wavelength dependence of the filter response exhibited anomolously large sidelobes for wavelengths shorter than $590 \mathrm{~nm}$. These discrepancies are probably due to chirp in the birefringence along the fiber.

How the seeded input was achieved is shown schematically in Fig. 2 A Soleil-Babinet compensator was used to change linear into elliptical polarization with a phase angle $\Delta \phi$ between major and minor axes. The beam was then focused onto the input end of the fiber, with the major axis of the input polarization tilted at an angle $\theta$ to a fiber polarization axis. The experiments were implemented with 30 ps pulses from a Coherent 740 dye laser of wavelength $590 \mathrm{~nm}$, pumped by a $Q$-switched, mode-locked Quantronix 416, doubled Nd:YAG laser. The two orthogonally polarized outputs were separated with a polarizing beamsplitter and detected on separate, slow photodiodes.

Typical results for self-switching $(R=0)$ are reproduced in Fig. 3. The two channels output start at 0 and $100 \%$, indicating that the fiber length is $L_{c}$ to within a few percent. The measured critical peak power is $750 \mathrm{~W}$, in good agreement with the calculated value of $713 \mathrm{~W}$ if the

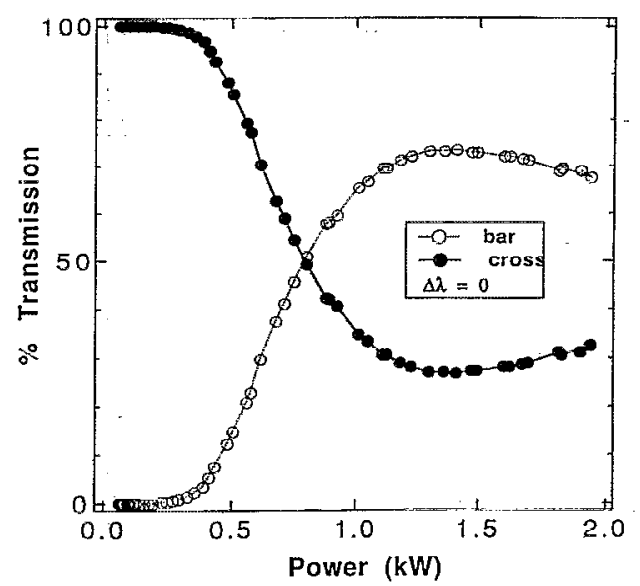

FIG. 3. All-optical switching between polarization states in a fiber filter tuned to the resonant wavelength. Power is input into the bar state only. SB and PB are the Soleil-Babinet compensator and the polarizing beam splitter, respectively.

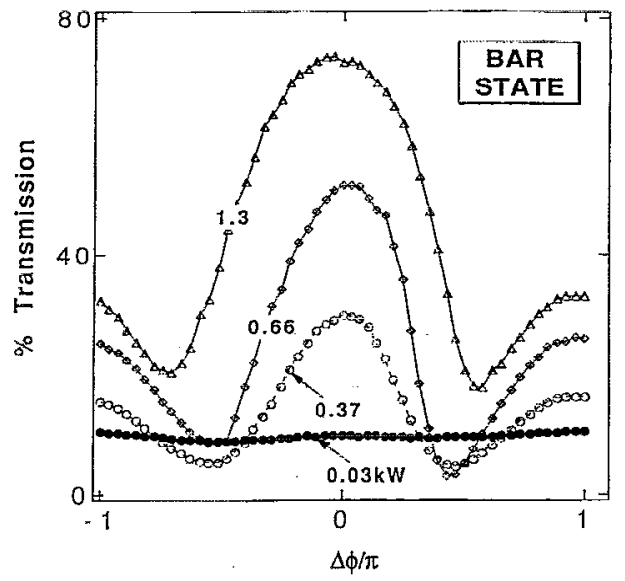

FIG. 4. Phase controlled all-optical switching of the bar state, with $10 \%$ of the input power in the control pulse. The four different curves correspond to successive larger input powers of $0.03,0.37,0.66$, and $1.3 \mathrm{KW}$.

effective coupling length of $127 \mathrm{~cm}$ is used. In excess of $70 \%$ is switched between the two polarization states, limited as usual by pulse break up. ${ }^{1,3}$ The onset of Stimulated Raman scattering leads to a flattening of the response above $1.1 \mathrm{~kW}$. The switching behavior was found to be very stable over the period of one day, the longest consecutive time the experiment was run.

The results of the seeded experiment for $R^{2}=0.1$ and variable $\Delta \phi$ are shown in Fig. 4 for four different input power levels. For $0.66 \mathrm{KW}$ input power, the output in the bar state (incidence polarization) can be switched from $>50 \%$ to $<5 \%$ by changing the relative phase from 0 to $\pi / 2$. The minimum in the bar state power at $\phi \simeq \pi / 2$ at the highest input power level also contains a stimulated Raman component and is actually deeper than indicated in Fig. 3. when the contributions due to stimulated Raman at the highest powers are taken into account, the agreement with theory is very good.

In order to obtain further insight into the mechanism operative here we studied the phase variation in the switch-

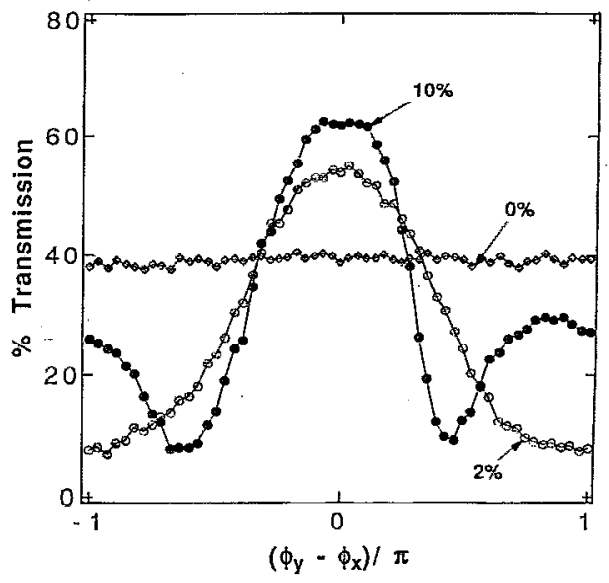

FIG. 5. Phase controlled $\left[\Delta \phi=\left(\phi_{y}-\phi_{x}\right) / \pi\right]$ all optical switching of the bar state with $750 \mathrm{~W}$ of input power in the signal pulse. The three different curves correspond to successive larger control beam powers of $0.0 \%, 0.02 \%$, and $0.1 \%$ of the input signal power. 
ing for different values of $R$. Shown in Fig. 5 are the results for the output bar state $\left[b_{x}\left(L_{c}\right)\right]$ with different amounts of seeding $\left[b_{p}(0)\right]$ and a fixed total input power in the signal $\left[b_{y}^{2}(0)+b_{y}^{2}=1050 \mathrm{~W}\right]$. Note that the changes with phase are progressively less when $R^{2}$ is reduced. Furthermore, when $R^{2}$ was reduced to zero (no control beam), the switching was essentially unaffected. These results, specifically the strong dependence on the seed power, lead us to conclude that this phase variation is unrelated to the switching predicted at the critical power for NLDCs. Instead this phase variation results from choosing the right initial conditions at the input end of the fiber, that is the details of the seeding of the second channel. Further calculations have shown that an initial detuning of the fiber filter from its resonance by using a different incident wavelength can improve further the switching characteristics. Experiments are currently underway to verify these predictions.

In summary, we have shown that by judiciously seeding the second input polarization of a rocking filter fiber, the all-optical switching response can be controlled by the phase of the seed beam. This opens new possibilities for implementing all-optical switching operations such as logic gates, etc. Finally, we note that a fixed phase relation between the pulses is required and timing jitter could smooth out the response measured here.

The research at the University of Central Florida was supported by AFOSR-90-086.

'S. R. Friberg, A. M. Weiner, Y. Silberberg, B. G. Sfez, and P. S. Smith, Opt. Lett. 13, 904 (1988).

${ }^{2}$ K. J. Blow, N. J. Doran, and B. K. Nayer, Opt. Lett. 14, 754 (1989).

${ }^{3}$ S. Trillo, S. Wabnitz, W. C. Banyai, N. Finlayson, C. T. Seaton, G. I. Stegeman, and R. H. Stolen, Appl. Phys. Lett. 53, 837 (1988); S. Trillo, S. Wabnitz, W. C. Banyai, N. Finlayson, C. T. Seaton, G. I. Stegeman, and R. H. Stolen, IEEE J. Quant. Electron. 25, 1042 (1989).

${ }^{4}$ M. N. Islam, C. D. Poole, and J. P. Gordon, Opt. Lett. 14, 1011 (1989).

${ }^{5}$ M. Morioka, M. Saruwatari, and A. Takada, Electron. Lett. 23, 453 (1987).

${ }^{6}$ R. Jin, J. P. Sokolof,, P. A. Harten, C. L. Chuang, S. G. Lee, M. Warren, H. M. Gibbs, N. Peyghambarian, J. N. Polky, and G. A. Pubanz, Appl. Phys. Lett. 56, 993 (1990).

${ }^{7}$ A. Miller, C. B. Park, and P. LiKamWa, Appl. Phys. Lett. 60, 97 (1992).

${ }^{8}$ J. S. Aitchison, A. H. Kean, C. N. Ironside, A. Villeneuve, and G. I. Stegeman, Electron. Lett. 27, 1709 (1991).

${ }^{9}$ S. Wabnitz, E. M. Wright, J. V. Moloney, C. T. Seaton, and G. I. Stegeman, Appl. Phys. Lett. 49, 838 (1986).

${ }^{10}$ R. H. Stolen, A. Ashkin, W. Pleibel, and J. M. Dziedzic, Opt. Lett. 9, 300 (1984). 